\title{
Endoscopic transnasal orbital decompression for Graves' ophthalmopathy, a systematic review of the literature*
}

\author{
Nikolaos Tsetsos ${ }^{1}$, Dimitrios Daskalakis',3, Dimitra Tzakri ${ }^{4}$, Sarantis Blioskas ${ }^{5}$, \\ John Goudakos' ${ }^{1}$ Konstantinos Markou ${ }^{6}$ \\ ' Department of Otorhinolaryngology-Head and Neck Surgery, 424 General Military Hospital, Thessaloniki, Greece \\ 2 Department of Otorhinolaryngology-Head and Neck Surgery, York Teaching Hospital, York, United Kingdom \\ ${ }^{3}$ Department of Otorhinolaryngology-Head and Neck Surgery, G. Gennimatas - St Demetrios Hospital, Thessaloniki, Greece \\ ${ }^{4}$ Department of Ophthalmology, 424 General Military Hospital, Thessaloniki, Greece \\ ${ }^{5}$ Department of Otorhinolaryngology-Head and Neck Surgery, Derriford Hospital, Plymouth, United Kingdom \\ ${ }^{6}$ 2nd Academic Otorhinolaryngology-Head and Neck Surgery Department, Aristotle University of Thessaloniki, Papageorgiou \\ Hospital, Thessaloniki, Greece
}

Rhinology 58: 1, 2 - 9, 2020

https://doi.org/10.4193/Rhin19.282

*Received for publication:

August 19, 2019

Accepted: September 21, 2019

\begin{abstract}
Background: The aim of this study was to assess all available data regarding the efficacy of endoscopic transnasal orbital decompression for Graves' ophthalmopathy.

Methodology: MEDLINE, ScienceDirect and the Cochrane Library databases as well as other sources were searched by two independent reviewers followed by extensive hand-searching for the identification of relevant studies. The primary outcome was the reduction in orbital proptosis. Secondary outcomes were the improvement in visual acuity, post-operative diplopia, and complications

Results: Twelve prospective and retrospective case series met the inclusion criteria. All of them demonstrated an improvement in postoperative proptosis that ranged from $2.07 \mathrm{~mm}$ to $8.2 \mathrm{~mm}$ (weighted mean improvement $5.05 \mathrm{~mm}$ ). Improvement in visual acuity was reported in all but one study. Studies presented a wide range of results regarding pre-existing and new-onset diplopia. Apart from diplopia, a wide variety of minor and major complications were noted in ten studies, the most serious of which being 3 cases of Cerebrospinal fluid (CSF) leak presented in 2 studies.
\end{abstract}

Conclusions: The present systematic review shows that endoscopic transnasal decompression safely addresses symptoms of Graves' ophthalmopathy. However, high-quality, large-sample, controlled studies need to be performed in the future.

Key words: endoscopic, Graves orbitopathy, decompression

\section{Introduction}

Graves' disease is an autoimmune multisystem disease in which $30 \%$ to $50 \%$ of patients develop an ophthalmopathy caused by enlargement of orbital fat and muscles leading to proptosis ${ }^{(1)}$. The mechanism of dysthyroid orbitopathy is unidentified and is speculated that thyroid-stimulating hormone receptor (TSHR) is expressed by fibroblasts in the orbital tissues. This results in gathering of T lymphocytes sensitized to antigens common in both thyroid cells and orbital contents and infiltration of the orbital fat and extraocular muscles with glycosaminoglycan, inflammatory cells, and immune complexes. This leads to an increase in the intraorbital contents and the consequent propto$\operatorname{sis}^{(2)}$. Although, thyroid eye disease often recedes and does not impair vision, however severe proptosis can lead to exposure keratopathy, diplopia and optic neuropathy ${ }^{(3)}$.

Dysthyroid orbitopathy seems to develop independently from the clinical course of the thyroid disease. Even if hyperthyroidism has been corrected patients may manifest ophthalmic 
findings at any time in the future ${ }^{(3)}$.

Myriad of medical treatments are used, including high-dose corticosteroids, radiotherapy and immunosuppressive agents such as cyclosporin. The steroids should be used in high doses for long treatment periods causing side-effects while radiotherapy despite being effective in alleviating optic neuropathy, does not always produce significant improvement in proptosis and cosmetic appearance. Indications for surgery have changed over the last years and although deterioration of visual acuity used to be the main indication, nowadays the interest has been shifted to cosmetic reasons as well ${ }^{(4)}$.

Dollinger first described orbital decompression for the treatment of patients with Graves' disease in 1911. Since then, various approaches have been adopted to decompress the orbit, by removing 1 to 4 of its bony walls ${ }^{(5)}$. In 1990, Kennedy et al. first reported the endoscopic transnasal medial and inferior wall decompression for thyroid eye disease. Performing this surgery via a transnasal route gives the advantage of clear visualization of orbital structures without any facial or oral scars rendering it an appealing choice, mainly due to aesthetic reasons ${ }^{(3)}$. Since then, most studies have reported results of mainly combined approaches and only a few have been conducted evaluating the effectiveness and the safety of purely endoscopic transnasal decompression for thyroid eye disease ${ }^{(6)}$.

In this systematic review we aimed to assess the outcomes of purely endoscopic transnasal orbital decompression collecting data from all available articles in the literature.

\section{Materials and methods}

This systematic review was performed according to the PRISMA 2009 checklist. For the purpose of this paper, original studies presenting outcomes of transnasal endoscopic orbital decompression were identified. No year limitation was imposed and both prospective and retrospective studies published in English were considered for inclusion.

MEDLINE, Science direct and the Cochrane Library electronic databases were searched from July 1st, 2019 until, August 1st 2019, in order to identify studies answering the research question. Additionally, clinicaltrials.gov and International Clinical Trials Registry Platform databases as well as the archives of major recent ENT conferences were also searched with the intention of identifying grey literature. The references of all retrieved studies and review papers were also carefully hand-searched.

Two reviewers (N.T. and D.D.) independently executed the comprehensive literature search. The following free-text terms were used for the MEDLINE database search: ["Graves disease" OR "Graves orbitopathy" OR "Graves ophthalmopathy" OR "Graves' disease" OR "Graves' orbitopathy" OR "Graves' ophthalmopathy" OR "thyroid eye disease" OR "thyroid ophthalmopathy" OR exophthalmos OR "dysthyroid orbitopathy"] AND [decompression] AND [orbit OR orbital] AND [endoscopic OR endoscopy].

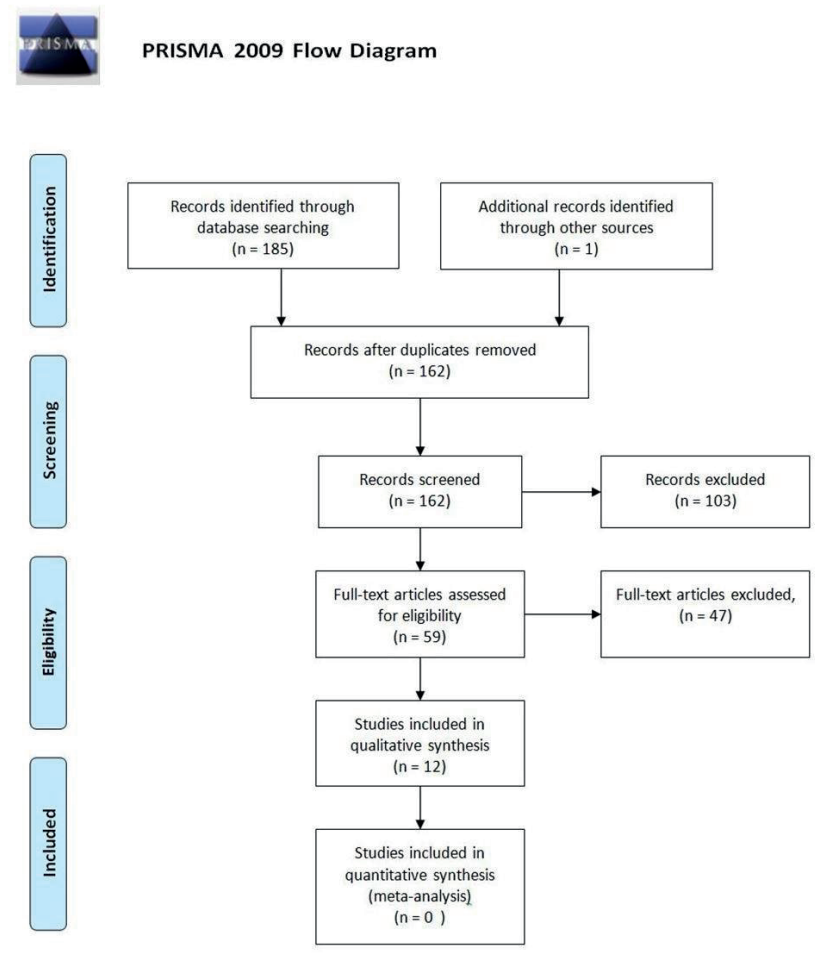

Figure 1. Literature search strategy. PRISMA flowchaii detailin literature search and review.

The search was restricted to humans and only articles published in English were included in the study. This search strategy was modified accordingly to suit the search rules of the other databases.

The study selection process was executed independently by the two reviewers and disagreements were resolved by consensus. The criteria for the inclusion and exclusion of studies in this systematic review were defined prior to commencing the literature search. Studies fulfilling the following criteria were eligible for inclusion: all randomized and nonrandomized controlled studies of surgical decompression were included, as well as prospective or retrospective case series of at least 10 decompressed orbits. Studies should include purely endoscopic transnasal approaches and any study evaluating combined approaches was excluded from our systematic review. Studies that assessed other techniques apart from endoscopic transnasal decompression were included only if they presented at least 10 purely endoscopic decompressions. Studies with revision endoscopic decompressions were also excluded.

The independent reviewers extracted data according to predetermined criteria. For this purpose a data-extraction form was designed prior to study examination and was used by the reviewers. Any arising disagreement was resolved through consensus. The following data was recorded from each of the eligible studies: general characteristics (first author, year of publication, country, study design, study sample, number of orbits decom- 
Table 1. Study characteristics.

\begin{tabular}{|c|c|c|c|c|c|c|c|c|c|c|}
\hline \multirow{2}{*}{$\begin{array}{l}\text { Lead } \\
\text { Author } \\
\text { (Year) ref }^{\text {Yea }}\end{array}$} & \multirow[t]{2}{*}{ Country } & \multirow[t]{2}{*}{ Study design } & \multicolumn{3}{|c|}{ Patients (No.) } & \multirow{2}{*}{$\begin{array}{l}\text { Number } \\
\text { of Orbits } \\
\text { Decom- } \\
\text { pressed }\end{array}$} & \multirow{2}{*}{$\begin{array}{l}\text { Follow- } \\
\text { up } \\
\text { (months) }\end{array}$} & \multirow[t]{2}{*}{ Indication (No) } & \multirow[t]{2}{*}{ End Points } & \multirow[t]{2}{*}{ Procedure } \\
\hline & & & Total & Male & $\begin{array}{l}\text { Fe- } \\
\text { male }\end{array}$ & & & & & \\
\hline $\begin{array}{l}\text { Neuge- } \\
\text { bauer et } \\
\text { al. } \\
(1996)^{6}\end{array}$ & Germany & $\begin{array}{l}\text { Retrospective } \\
\text { case series } \\
\text { study }\end{array}$ & 18 & 2 & 16 & 36 & $\begin{array}{l}\frac{1}{2} \\
\text { and } 4 \\
\text { to } 6\end{array}$ & ${ }^{*} \mathrm{CON}$ & $\begin{array}{l}\text { †PR, }{ }^{\mp} \mathrm{VAl} \text {, } \\
\text { Change in } \\
\text { angle of squint, } \\
\text { vertical gaze } \\
\text { motility \& ad- } \\
\text { duction, }{ }^{\S} \mathrm{POC}\end{array}$ & "MIWR \\
\hline $\begin{array}{l}\text { Lund et al. } \\
(1997)^{4}\end{array}$ & UK & $\begin{array}{l}\text { Retrospective } \\
\text { case series } \\
\text { study }\end{array}$ & 13 & 1 & 12 & 24 & 55.4 & $\begin{array}{l}\text { Proptosis (13) } \\
\text { Diplopia (7) } \\
\text { CON (3) } \\
\text { Reduced \#VA (3) and } \\
\text { colour vision (3) }\end{array}$ & $\begin{array}{l}\text { PR } \\
\text { VA and color } \\
\text { vision Improve- } \\
\text { ment } \\
\text { *POD \& POC }\end{array}$ & MIWR \\
\hline $\begin{array}{l}\text { Koay et al. } \\
(1997)^{12}\end{array}$ & UK & $\begin{array}{l}\text { Case series } \\
\text { study }\end{array}$ & 15 & 5 & 10 & 30 & 3 to 6 & $\begin{array}{l}\operatorname{CON}(2), ` E K(2) \text { acute } \\
\text { proptosis (1) congestive } \\
\text { symptoms (10) }\end{array}$ & $\begin{array}{l}\text { PR } \\
\text { POD \& POC }\end{array}$ & MIWR \\
\hline $\begin{array}{l}\text { Michel et } \\
\text { al. }(2001)^{8}\end{array}$ & Germany & $\begin{array}{l}\text { Retrospective } \\
\text { case series } \\
\text { study }\end{array}$ & 78 & 15 & 63 & 145 & 41.5 & $\begin{array}{l}\text { CON (61), corneal invol- } \\
\text { vement (3), extraocular } \\
\text { muscle involvement (6), } \\
\text { corneal and extraocular } \\
\text { muscle involvement (7), } \\
\text { cosmesis (1) }\end{array}$ & $\begin{array}{l}\text { PR, VAI } \\
\text { Post op Ocular } \\
\text { Motility } \\
\text { POD \& POC }\end{array}$ & MIWR \\
\hline $\begin{array}{l}\text { Yuen et al. } \\
(2002)^{9}\end{array}$ & China & $\begin{array}{l}\text { Retrospective } \\
\text { case series } \\
\text { study }\end{array}$ & 14 & 7 & 7 & 23 & 32 & $\begin{array}{l}\text { CON and/or EK } \\
\text { or with other severe } \\
\text { symptoms (17) } \\
\text { cosmesis (4) }\end{array}$ & $\begin{array}{l}\text { PR, VAI } \\
\text { Change in } \\
\text { retraction of } \\
\text { the upper and } \\
\text { lower eyelids } \\
\text { and intraocular } \\
\text { pressure } \\
\text { POD \& POC }\end{array}$ & MIWR \\
\hline $\begin{array}{l}\text { Stiglmayer } \\
\text { et al. } \\
(2004)^{10}\end{array}$ & Croatia & $\begin{array}{l}\text { Case series } \\
\text { study }\end{array}$ & 21 & 8 & 13 & 32 & 12.5 & $\begin{array}{l}\text { CON and/or EK } \\
\text { or with other severe } \\
\text { symptoms (17) } \\
\text { cosmesis (4) }\end{array}$ & $\begin{array}{l}\text { PR, VAI } \\
\text { Change in } \\
\text { retraction of } \\
\text { the upper and } \\
\text { lower eyelids } \\
\text { and intraocular } \\
\text { pressure } \\
\text { POD \& POC }\end{array}$ & *MWR \\
\hline $\begin{array}{l}\text { Kasper- } \\
\text { bauer et } \\
\text { al. } \\
(2005)^{11}\end{array}$ & USA & $\begin{array}{l}\text { Retrospective } \\
\text { case series } \\
\text { study }\end{array}$ & 59 & 18 & 41 & 88 & 1.5 to 2 & $\begin{array}{l}\text { EK (59) } \\
\text { Decompression in pre- } \\
\text { paration for eye muscle } \\
\text { surgery (27) } \\
\text { Diplopia (37) } \\
\text { CON (16) }\end{array}$ & $\begin{array}{l}\text { PR } \\
\text { Need for } \\
\text { further opht- } \\
\text { halmologic } \\
\text { procedures } \\
\text { POD \& POC } \\
\text { length of hos- } \\
\text { pital stay }\end{array}$ & MIWR \\
\hline $\begin{array}{l}\text { She et al. } \\
(2012)^{12}\end{array}$ & Taiwan & $\begin{array}{l}\text { Retrospective } \\
\text { case series } \\
\text { study }\end{array}$ & 25 & 14 & 11 & 42 & 1 and 3 & $\begin{array}{l}\text { severe/acute proptosis } \\
\text { (13) CON ( } 7) \\
\text { proptosis and decre- } \\
\text { ased VA (4), severe EK } \\
\text { (1) }\end{array}$ & $\begin{array}{l}\text { PR } \\
\text { VAI } \\
\text { POD \& POC }\end{array}$ & MIWR \\
\hline $\begin{array}{l}\text { Lal et al. } \\
(2013)^{13}\end{array}$ & India & $\begin{array}{l}\text { Case series } \\
\text { study }\end{array}$ & 12 & 10 & 2 & 24 & At least 6 & $\begin{array}{l}\text { Proptosis with } \\
\text { corneal exposure (8) } \\
\text { cosmesis (2) } \\
\text { CON (2) }\end{array}$ & $\begin{array}{l}\text { PR } \\
\text { VA Improve- } \\
\text { ment } \\
\text { POD \& POC }\end{array}$ & MIWR \\
\hline $\begin{array}{l}\text { Gulati et } \\
\text { al. } \\
(2015)^{14}\end{array}$ & Norway & $\begin{array}{l}\text { Retrospective } \\
\text { case series } \\
\text { study }\end{array}$ & 37 & 3 & 34 & 66 & 46 & NR & $\begin{array}{l}\text { PR, VAI } \\
\text { Post-op strabis- } \\
\text { mus, restricted } \\
\text { motility } \\
\text { POD \& POC }\end{array}$ & $\begin{array}{l}\operatorname{MWR}(6) \\
\operatorname{MIWR}(31)\end{array}$ \\
\hline
\end{tabular}




\begin{tabular}{|c|c|c|c|c|c|c|c|c|c|c|}
\hline \multirow{2}{*}{$\begin{array}{l}\text { Lead } \\
\text { Author } \\
\text { (Year) }{ }^{\text {ref }}\end{array}$} & \multirow[t]{2}{*}{ Country } & \multirow[t]{2}{*}{ Study design } & \multicolumn{3}{|c|}{ Patients (No.) } & \multirow{2}{*}{$\begin{array}{l}\text { Number } \\
\text { of Orbits } \\
\text { Decom- } \\
\text { pressed }\end{array}$} & \multirow{2}{*}{$\begin{array}{l}\text { Follow- } \\
\text { up } \\
\text { (months) }\end{array}$} & \multirow[t]{2}{*}{ Indication (No) } & \multirow[t]{2}{*}{ End Points } & \multirow[t]{2}{*}{ Procedure } \\
\hline & & & Total & Male & $\begin{array}{l}\text { Fe- } \\
\text { male }\end{array}$ & & & & & \\
\hline $\begin{array}{l}\text { Lv et al. } \\
(2015)^{15}\end{array}$ & China & $\begin{array}{l}\text { Retrospective } \\
\text { case series } \\
\text { study }\end{array}$ & 43 & 12 & 31 & 72 & 9 & $\mathrm{CON}$ & $\begin{array}{l}\text { PR } \\
\text { VAI } \\
\text { POD \& POC }\end{array}$ & MWR \\
\hline $\begin{array}{l}\text { Wu et al. } \\
(2015)^{16}\end{array}$ & China & $\begin{array}{l}\text { Retrospective } \\
\text { case series } \\
\text { study }\end{array}$ & 108 & 34 & 74 & 216 & 12 to 24 & $\begin{array}{l}\text { Type } 1 \text { Graves' } \\
\text { orbitopathy }\end{array}$ & $\begin{array}{l}\text { PR } \\
\text { Mean surgical } \\
\text { time } \\
\text { POD \& POC }\end{array}$ & MWR \\
\hline
\end{tabular}

${ }^{*} \mathrm{CON}$ : Compressive Optic Neuropathy, ${ }^{\dagger}$ Proptosis Reduction, ${ }^{\ddagger}$ VAI: Visual Acuity Improvement, ${ }^{5}$ POC: Post-Operative Complications, " MIWD: Medial \& Inferior Wall Removal, " VA: Visual Acuity, *POD: Post-Operative Diplopia, •EK: Exposure Keratitis, " MWD: Medial Wall Removal.

pressed, indication for surgery and length of follow-up) and outcome data (primary and secondary outcomes).

The primary outcome was the reduction in orbital proptosis. Secondary outcomes were the improvement in visual acuity, post-operative diplopia and complications. Safety was assessed on the basis of major and minor adverse events reported in the studies. If available, statistical data, such as confidence intervals, standard deviations, p-values etc. were reported; otherwise, results were presented narratively.

\section{Results}

A total of 185 studies were retrieved through the systematic search of bibliographic databases. One additional record from other source, was also identified. After removing duplicates, 162 papers were eligible for title-abstract screening. Finally, twelve articles providing data able to answer the research question were identified $^{(4,6-16)}$. None of the twelve aforementioned studies were eligible for qualitative synthesis. The flow diagram is depicted in Figure 1. All included studies were written in English and published from 1996 to 2015 . A total of 443 patients $(29,1 \%$ males and $70,9 \%$ females) were recruited, with a total of 798 endoscopic transnasal orbital decompressions having been performed. Study characteristics are presented in Table 1.

Proptosis reduction was assessed in all included studies. Pre and post-operative ocular protrusion examination was included Hertel exophthalmometry in all studies except for one that did not report their method of assessment ${ }^{(4)}$. The basis of exophthalmometry is the measure of the distance of the corneal apex from the level of the lateral orbital rim using an exophthalmometer ${ }^{(17)}$. All of them demonstrated an improvement in postoperative proptosis that ranged from $2.07 \mathrm{~mm}^{(12)}$ to $8.2 \mathrm{~mm}^{(16)}$. Overall, weighted mean in proptosis reduction estimated at $5.05 \mathrm{~mm}$ (Table 2).

Change in visual acuity was evaluated in all studies aside from two ${ }^{(11,16)}$. All studies demonstrated an improvement in postoperative visual acuity apart from one $\mathrm{e}^{(13)}$. In this study, a patient that had endoscopic decompression for rapidly progressive visual loss with presumed optic nerve compression demonstrated significant improvement of vision in the eye with partial visual loss, but no improvement was noted in the eye with complete visual loss. No other data were presented about visual acuity changes in the patients who were operated for other indications (Table 2).

Diplopia was discussed in all but one study ${ }^{(6)}$. Studies presented a wide range of results regarding pre-existing and new onset diplopia. The largest percentage of pre-existing diplopia was $83.3 \%$ which increased post operatively due to the addition of a new onset diplopia ${ }^{(12)}$. Similar results were presented in the 2 nd largest study ${ }^{(8)}$ with the percentage of post-operative diplopia being $81.3 \%$ while transient new onset diplopia was invariable in all cases with binocular vision and resolved over the next two to eight weeks without squint surgery ${ }^{(13)}$.

Apart from diplopia, two of the included studies did not mention any other intra or post-operative complication ${ }^{(7,9)}$. All remaining studies presented a wide variety of minor and major intra and post-operative complications. A total number of 29 patients experienced post-operative epistaxis making it, overall, the most common complication ${ }^{(15,16)}$. The most serious complications were three cases of Cerebrospinal fluid (CSF) leak that presented in two studies ${ }^{(11,12)}$. Two of these cases were identified and managed at the time of decompression ${ }^{(11)}$ while the third case had a surgical re-exploration on the seventh postoperative day due to persistent clear nasal discharge ${ }^{(12)}$. Taking into account the complexity of post-operative frontal sinusitis, it is worth mentioning that two cases of this complication were noted in two different studies ${ }^{(8,13)}$. The first case was reported two months post-operatively ${ }^{(8)}$ while the second appeared four months after the decompression receding with conservative treatment ${ }^{(13)}$. Apart from frontal sinusitis, six more cases of postoperative sinusitis were reported in three studies $(8,11,14)$. Only one patient was treated conservatively ${ }^{(14)}$, while the remaining five needed endoscopic sinus surgery. Furthermore, only one patient without a previous history of sinus disease developed an ethmoid mucocele that required endoscopic drainage ${ }^{(11)}$. At last, 
Table 2. Primary and secondary outcomes of included studies.

\begin{tabular}{|c|c|c|c|c|c|c|c|c|}
\hline \multirow{2}{*}{$\begin{array}{l}\text { Lead Author } \\
\text { (Year) }\end{array}$} & \multirow{2}{*}{$\begin{array}{l}\text { Mean proptosis } \\
\text { reduction }(\mathrm{mm})\end{array}$} & \multicolumn{3}{|c|}{ Change in visual acuity } & \multicolumn{3}{|c|}{ Diplopia } & \multirow{2}{*}{$\begin{array}{l}\text { Post-operative } \\
\text { complications (no) }\end{array}$} \\
\hline & & $\begin{array}{l}\text { Re- } \\
\text { port- } \\
\text { ed }\end{array}$ & $\begin{array}{l}\text { Not } \\
\text { re- } \\
\text { port- } \\
\text { ed }\end{array}$ & Comment & $\begin{array}{l}\text { Re- } \\
\text { port- } \\
\text { ed }\end{array}$ & $\begin{array}{l}\text { Not } \\
\text { re- } \\
\text { port- } \\
\text { ed }\end{array}$ & Comment & \\
\hline $\begin{array}{l}\text { Neugebaur et al. } \\
\text { (1996) }\end{array}$ & $\begin{array}{l}\text { Group } 1(n=9) \text { : pre- } \\
\text { op 23(SD 5) post-op } \\
\text { 20(SD 4) Group } \\
\text { 2(n=9): pre-op } \\
\text { 23(SD 4.5) post-op } \\
20.5 \text { (SD4.5) } \\
\text { Overall: } 3\end{array}$ & $\checkmark$ & & $\begin{array}{l}\text { Mean group } 1 \text { ( } n= \\
\text { 14): pre-op } 0.35 \text { (SD } \\
0.26) \text {, post-op } 0.59 \\
\text { (SD 0.2, Mean group } \\
2(n=13) \text { : pre-op } 0.6 \\
\text { (SD 0.18), post-op } \\
0.7 \text { (SD 0.16) }\end{array}$ & & $\checkmark$ & & $\begin{array}{l}\text { Mild infraorbital } \\
\text { hypoesthesia that } \\
\text { receded some days } \\
\text { later }\end{array}$ \\
\hline $\begin{array}{l}\text { Lund et al. } \\
\text { (1997) }\end{array}$ & $\begin{array}{l}4.4 \\
\text { (range 1-10) }\end{array}$ & $\checkmark$ & & & $\checkmark$ & & $\begin{array}{l}4 \text { cases requiring } \\
\text { surgery }\end{array}$ & $\begin{array}{l}\text { Epiphora not re- } \\
\text { quired surgery (1) }\end{array}$ \\
\hline $\begin{array}{l}\text { Koay et al. } \\
\text { (1997) }\end{array}$ & $\begin{array}{l}3.9 \\
\text { (range 2-8) }\end{array}$ & $\checkmark$ & & $\begin{array}{l}\text { Improved in the two } \\
\text { patients with com- } \\
\text { pressive neuropathy }\end{array}$ & $\checkmark$ & & $\begin{array}{l}2 \text { cases with pre-op } \\
\text { diplopia: minimal } \\
\text { post-op changes. } \\
\text { Both needed cor- } \\
\text { rective strabismus } \\
\text { surgery. }\end{array}$ & $\begin{array}{l}\text { No major intra or } \\
\text { post-operative } \\
\text { complications }\end{array}$ \\
\hline $\begin{array}{l}\text { Michel et al. } \\
(2001)\end{array}$ & $\begin{array}{l}3.94 \pm 2.73 \\
\text { (range }-1-11)\end{array}$ & $\checkmark$ & & $\begin{array}{l}\text { Increased from a } \\
\text { preoperative avera- } \\
\text { ge of } 0.50 \pm 0.27 \\
\text { (range, } 0.01-1.25 \text { ) to } \\
0.75 \pm 0.21 \text { (range, } \\
0.01-1.25 \text { ) postope- } \\
\text { ratively }\end{array}$ & $\checkmark$ & & $\begin{array}{l}81.2 \% \text { post op } \\
\text { diplopia }\end{array}$ & $\begin{array}{l}\text { maxillary sinusitis } \\
\text { (needed surgery) (1) } \\
\text { frontal sinusitis } 2 \\
\text { months post-op ( } 1 \text { ) } \\
\text { Persistent infraorbi- } \\
\text { tal nerve compro- } \\
\text { mise (4) }\end{array}$ \\
\hline $\begin{array}{l}\text { Yuen et al. } \\
(2002)\end{array}$ & $\begin{array}{l}4.6 \\
\text { (range 1-8) }\end{array}$ & $\checkmark$ & & $\begin{array}{l}\text { Improved for } 16 \\
\text { (70\%) eyes with a } \\
\text { median improve- } \\
\text { ment of } 3 \text { Snellen } \\
\text { lines (range, 1-8 } \\
\text { lines) }\end{array}$ & $\checkmark$ & & $\begin{array}{l}1 \text { case of not im- } \\
\text { proved pre-existed } \\
\text { diplopia } \\
5 \text { new-onset cases } \\
\text { All corrected with } \\
\text { surgery }\end{array}$ & $\begin{array}{l}\text { No other complica- } \\
\text { tions }\end{array}$ \\
\hline $\begin{array}{l}\text { Stiglmayer et al. } \\
(2004)\end{array}$ & $\begin{array}{l}4.6 \pm 1.7 \\
(p<0.001)\end{array}$ & $\checkmark$ & & $\begin{array}{l}\text { Improved from } \\
\text { pre-op } 0.81 \pm 0.28 \\
\text { (mean } \pm S D \text { ) to } \\
\text { post- op } 0.92 \pm 0.21 \\
(p=0.0032)\end{array}$ & $\checkmark$ & & $\begin{array}{l}11 \text { cases pre-existed } \\
\text { in } 2 \text { resolved and in } \\
9 \text { persisted } \\
\text { New-onset diplopia } \\
\text { in } 8 \text { patients }\end{array}$ & $\begin{array}{l}\text { Deterioration of } \\
\text { ocular motility } \\
\text { disturbance } \\
\text { (18 eyes) } \\
\text { slight intraoperative } \\
\text { bleeding ( } 3 \text { ) } \\
\text { post-op periorbital } \\
\text { hematoma ( } 1 \text { ) }\end{array}$ \\
\hline $\begin{array}{l}\text { Kasperbauer et al. } \\
(2010)\end{array}$ & $\begin{array}{l}2.5 \\
\text { [Right: } 2.4 \\
\text { (range:-2.5-10.0) } \\
\text { Left: } 2.5 \\
\text { (range(-6.0-7.0] }\end{array}$ & & $\checkmark$ & & $\checkmark$ & & $\begin{array}{l}\text { New diplopia } \\
\text { requiring strabismus } \\
\text { surgery developed } \\
\text { in } 7 \text { of } 22(32 \%) \\
\text { patients }\end{array}$ & $\begin{array}{l}{ }^{*} \text { CSF leaks managed } \\
\text { intraoperatively ( } 2 \text { ) } \\
\text { Post-op sinusitis } \\
\text { required revision } \\
\text { antrostomies (2) } \\
\text { ethmoid mucocele } \\
\text { required endoscopic } \\
\text { drainage (1) }\end{array}$ \\
\hline $\begin{array}{l}\text { She et al. } \\
(2012)\end{array}$ & $\begin{array}{l}1.93 \pm 0.25 \\
(\text { range } 0.5-6) \\
1 \text { month post-op } \\
\text { and } \\
2.07 \pm 0.29 \\
\text { (range } 0-7) \\
3 \text { months post-op } \\
(p<0.01)\end{array}$ & $\checkmark$ & & $\begin{array}{l}\text { Increased from a } \\
\text { preoperative avera- } \\
\text { ge of } 0.45 \pm 0.34 \text { to } \\
0.66 \pm 0.36 \text { and } 0.70 \\
\pm 0.35 \text { after } 1 \text { and } 3 \\
\text { months post-op }\end{array}$ & $\checkmark$ & & $\begin{array}{l}20 \text { patients ( } 35 \\
\text { orbits, } 83.3 \% \text { ) pre- } \\
\text { existed } \\
\text { All persisted post- } \\
\text { op } \\
1 \text { new-onset (two } \\
\text { orbits) }\end{array}$ & $\begin{array}{l}\text { Small } \\
\text { CSF leak (1) }\end{array}$ \\
\hline $\begin{array}{l}\text { Lal et al. } \\
\text { (2013 }\end{array}$ & 3.70 (range 2-6) & $\checkmark$ & & $\begin{array}{l}\text { Significant improve- } \\
\text { ment in the eye with } \\
\text { partial visual loss, } \\
\text { no improvement in } \\
\text { the eye with com- } \\
\text { plete visual loss }\end{array}$ & $\checkmark$ & & $\begin{array}{l}\text { new-onset diplopia } \\
\text { invariable in all } \\
\text { cases, resolved } \\
\text { over the next } 2 \text { to } 8 \\
\text { weeks }\end{array}$ & $\begin{array}{l}\text { Persistent frontal } \\
\text { sinusitis (1) }\end{array}$ \\
\hline
\end{tabular}




\begin{tabular}{|c|c|c|c|c|c|c|c|c|}
\hline \multirow{2}{*}{$\begin{array}{l}\text { Lead Author } \\
\text { (Year) }\end{array}$} & \multirow{2}{*}{$\begin{array}{l}\text { Mean proptosis } \\
\text { reduction }(\mathrm{mm})\end{array}$} & \multicolumn{3}{|c|}{ Change in visual acuity } & \multicolumn{3}{|c|}{ Diplopia } & \multirow{2}{*}{$\begin{array}{l}\text { Post-operative } \\
\text { complications (no) }\end{array}$} \\
\hline & & $\begin{array}{l}\text { Re- } \\
\text { port- } \\
\text { ed }\end{array}$ & $\begin{array}{l}\text { Not } \\
\text { re- } \\
\text { port- } \\
\text { ed }\end{array}$ & Comment & $\begin{array}{l}\text { Re- } \\
\text { port- } \\
\text { ed }\end{array}$ & $\begin{array}{l}\text { Not } \\
\text { re- } \\
\text { port- } \\
\text { ed }\end{array}$ & Comment & \\
\hline $\begin{array}{l}\text { Gulati et al. } \\
\text { (2015) }\end{array}$ & 4.0 & $\checkmark$ & & $\begin{array}{l}\text { Improved from } \\
\text { a median value } \\
\text { (range) of } 0.8 \\
(0.05-1.25) \text { to } 1.0 \\
(0.4-1.25) \\
(p=0.006)\end{array}$ & $\checkmark$ & & $\begin{array}{l}\text { Seventeen patients } \\
\text { (46\%) pre-existed } \\
7 \text { cases of new- } \\
\text { onset diplopia (19\%) } \\
8 \text { cases of worsened } \\
\text { diplopia ( } 22 \%)\end{array}$ & $\begin{array}{l}\text { Delayed sinusitis } \\
\text { postoperatively (3) }\end{array}$ \\
\hline $\begin{array}{l}\text { Z. Lv et al. } \\
\text { (2015) }\end{array}$ & $\begin{array}{l}6.2 \pm 1.2 \\
(p<0.001)\end{array}$ & $\checkmark$ & & $\begin{array}{l}\text { Improvement from } \\
-0.65 \pm 0.30 \text { to }-0.25 \\
\pm 0.22, \text { post-op, with } \\
\text { a mean improve- } \\
\text { ment of } 0.55 \pm 0.17 \\
(p<0.001)\end{array}$ & $\checkmark$ & & $\begin{array}{l}5 \text { cases of new- } \\
\text { onset diplopia post } \\
\text { op with complete } \\
\text { resolution within } 3 \\
\text { months } \\
2 \text { cases of deteriora- } \\
\text { tion in their pre-ex }\end{array}$ & $\begin{array}{l}\text { Post-op epistaxis } \\
\text { all resolved without } \\
\text { nasal packing (10) }\end{array}$ \\
\hline $\begin{array}{l}\text { Wu et al. } \\
\text { (2015) }\end{array}$ & $\begin{array}{l}8.2 \pm 1.8 \\
\text { (range 4-11) }\end{array}$ & & $\checkmark$ & & $\checkmark$ & & $\begin{array}{l}25 \text { cases ( } 23.1 \%) \text { of } \\
\text { new-onset diplopia } \\
\text { with complete reso- } \\
\text { lution in } 23 \text { of these } \\
\text { within } 3 \text { months, } 2 \\
\text { patients required } \\
\text { squint surgery. }\end{array}$ & $\begin{array}{l}\text { Post-op epistaxis } \\
\text { (19) } \\
\text { intraorbital hemor- } \\
\text { rhages ( } 2 \text { ) } \\
\text { orbital emphysema } \\
\text { (3) }\end{array}$ \\
\hline
\end{tabular}

${ }^{*}$ CSF: Cerebrospinal Fluid.

there was no report of perioperative death or post-operative vision loss in any study.

Although the aim of our study was to proceed to a meta-analysis, no quantitative synthesis could be performed, due to the absence of data, key statistical measures (standard deviation) and/or the variable values, out of which such measures could be extrapolated.

\section{Discussion}

A myriad of different techniques and approaches have been reported for the treatment of Graves' ophtalmophaty ${ }^{(18)}$. In 1957, Walsh and Ogura described the transantral decompression, which has been the most common and preferred technique for decades ${ }^{(19)}$. Removal of the entire orbital floor is achieved after a maxillary antrostomy while trying to keep intact the infraorbital nerve. Lamina papyracea is exposed and removed through a transantral ethmoidectomy. Despite avoiding an external incision this approach is linked to morbidity of a Caldwell-Luc procedure. This includes considerable oedema and discomfort immediately postoperatively combined with persistent numbness over the mandibular region. The Walsh-Ogura procedure achieves an average of 4 to $6 \mathrm{~mm}$ of reduction in proptosis. The advent of endoscopic transnasal approach by Kennedy et al. has rendered external approaches obsoletes ${ }^{(3)}$.The advantages of this procedure are; decreased morbidity compared to external ethmoidectomy or transantral surgery, also less dysesthesia of the infraorbital nerve, better cosmetic results with less intraoperative blood loss and shorter length of inpatient stay ${ }^{(20)}$.
Weighted mean in proptosis reduction among the twelve included studies was estimated at $5.05 \mathrm{~mm}$. The lowest percentage of proptosis reduction across the included studies was observed in a series of 42 orbital decompressions. In this study patients were examined one and three months post-operatively with no statistically significant change between these follow-ups ${ }^{(12)}$. On the contrary, the highest percentage was shown in the largest series (216 decompressions). However, one should bear in mind that all patients included in this study suffered only from type 1 Graves orbitopathy who characteristically have the best response to decompressive surgery regardless of the type of surgical intervention ${ }^{(16)}$. This variation can be explained by several factors, such as the degree of preoperative exophthalmos, amount of bone removed, indications for surgery and different follow-up times.

Overall, endoscopic decompression seems to have the advantage of improving visual acuity in most cases. However, a variation in results among individuals is present in most of the studies. This wide variation in results most likely reflects the heterogeneous patient population and different indications for surgery. It can be argued that whenever cosmesis is the main indication for surgery, small changes in visual acuity could be explained by normal or near-normal pre-operative measurements ${ }^{(18)}$. Most studies did not weigh results according to the indication for surgery, therefore interpretation of the results must be done cautiously. Additionally, most possibly, a long history of optic nerve compression could explain poorer results post-operatively due to permanent damage of the optic nerve. So, it is problema- 
tic to compare the results among patients with totally different indications for decompression.

Endoscopic orbital decompression avoids the need for an intraoral incision and provides the surgeon with excellent visualization of the region of the ethmoid roof and orbital apex. Removal of the lamina papyracea and medial orbital floor, along with the underlying periorbital fascia, enables the enlarged orbital muscles and fat to decompress into the ethmoid and maxillary sinuses. This decompression allows the bulb of the eye to recess back into the orbit. However, with ocular recession the vector of pull for the extraocular muscles changes and postoperative double vision may result ${ }^{(22)}$. The surgical methods advocated to avoid diplopia are 1) preservation of a horizontal bony strut at the junction of the medial wall and floor of the orbit (Inferomedial Orbital Strut)(23), 2) "balanced decompression" involving removal of both medial and lateral orbital walls so as to avoid the displacement of the orbit in any direction ${ }^{(24,25)}$, and 3) the orbital sling technique, which makes use of a horizontal strip of periorbital fascia to prevent prolapse of the medial rectus muscle ${ }^{(22)}$. The endoscopic approach does not allow access to the lateral orbital wall, thus making it impossible to undertake the "balanced decompression" technique by a pure endoscopic approach.

The results of this systematic review are liable to certain limitations. First, although our aim was to include randomized controlled trials for the sake of the greater power of our research, we did not manage to retrieve anyone in our thorough literature search. On the contrary, only retrospective or prospective case series with no control group were found in the literature. The lack of control group made it impossible to draw safe conclusions regarding the inferiority or the superiority of endoscopic transnasal over older traditional techniques. Additionally, not only did we include low evidence and high risk of bias case series but we had difficulty in presenting results from studies that were characterized by low sample sizes, incomplete reporting data in most of them in combination with the heterogeneity among study designs and indications for surgery. Furthermore, we conducted a comprehensive search with language limitation in several bibliographic databases and the probability to have missed potential eligible studies is not to be underestimated.

\section{Conclusion}

It seems that the transnasal endoscopic technique is safe and effective in reducing proptosis as well as improving visual acuity. Although there are favorable cosmetic results and rare complications, the risk of deteriorating pre-existing or causing new onset of diplopia requiring squint surgery is still considerable. Larger, high quality, controlled studies ideally with subgroup of patients undergoing modified transanasal endoscopic decompressions (sling technique, preservation of Inferomedial Orbital Strut, "balanced decompression") are required so that safe conclusions can be derived.

\section{Acknowledgements}

This study was not supported by any company or persons other than the authors.

\section{Authorship contribution}

NT: Literature search, data extraction, analysis and manuscript production, DD: Design, analysis and manuscript review, DT: Data extraction and analysis, SB: Manuscript review and preparation, JG: Manuscript review and preparation; KM: Design and expert manuscript review.

\section{Conflict of interest}

All authors declare that there are no conflicts of interest to be reported.

\section{References}

1. Platt MP, Sindwani R, Metson R. Endoscopic orbital decompression. Oper Tech Otolaryngol Neck Surg. 2008;19(3):162-166.

2. Leong SC, White PS. Outcomes following surgical decompression for dysthyroid orbitopathy (Graves's disease). Curr Opin Otolaryngol Head Neck Surg. 2010;18(1):3743.

3. Kennedy DW, Goodstein ML, Miller NR Zinreich SJ. Endoscopic transnasal orbita decompression. Arch Otolaryngol Head Neck Surg. 1990;116(3):275-282.

4. Lund VJ, Larkin G, Fells P, Adams G. Orbital decompression for thyroid eye disease: a comparison of external and endoscopic techniques. J Laryngol Otol. 1997:111(11):1051-1055.

5. Yao WC, Sedaghat AR, Yadav P, Fay A, Metson R. Orbital Decompression in the
Endoscopic Age: The Modified Inferomedial Orbital Strut. Otolaryngol Head Neck Surg. 2016;154(5):963-969.

6. Neugebauer A, Nishino K, Neugebauer P, Konen W, Michel O. Effects of bilateral orbital decompression by an endoscopic endonasal approach in dysthyroid orbitopathy. Br J Ophthalmol. 1996;80(1):58-62.

7. Koay B, Bates G, Elston J.Endoscopic orbital decompression for thyroid eye disease. Eye. 1998;12(6):990-995.

8. Michel O, Oberla N, Neugebauer P Neugebauer A, Ru W. Follow-up of Transnasal Orbital Decompression in Severe Graves. Ophthalmology. 2001;6420(00):400404

9. Yuen APW, Kwan KYW, Chan E, Kung AWC, Lam KSL. Endoscopic transnasal orbital decompression for thyrotoxic orbitopathy. Hong Kong Med J. 2002;8(6):406-410.
10. Stiglmayer N, Mladina R, Tomiæ M, Tojagiæ $M$, Juri J, Bubaš $N$, et al. Endonasal endoscopic orbital decompression in patients with Graves' ophthalmopathy. Croat Med J. 2004 Jun;45(3):318-22.

11. Kasperbauer JL, Hinkley L. Endoscopic orbital decompression for Graves' ophthalmopathy. Am J Rhinol. 19(6):603-606.

12. She Y-Y, Chi C-C, Chu S-T. Transnasal endoscopic orbital decompression: 15-year clinical experience in Southern Taiwan. J Formos Med Assoc. 2014;113(9):648-655.

13. Lal P, Thakar A, Tandon N. Endoscopic orbital decompression for Graves 'orbitopathy. Indian J Endocrinol Metab. 2013;17(2).

14. Gulati S, Ueland H, Haugen O, Haugen O, Danielsen A, Rødahl E.Long-term follow-up of patients with thyroid eye disease treated with endoscopic orbital decompression. Acta Ophthalmol. 2015 Mar;93(2):178-83. 
15. Lv Z, Selva D, Yan W, Daniel P, Tu Y, Wu W Endoscopical Orbital Fat Decompression with Medial Orbital Wall Decompression for Dysthyroid Optic Neuropathy. Curr Eye Res. 2016;41(2):150-158

16. Wu W, Selva D, Bian $Y$, Wang $X$, Sun $M$, Kong $Q$, et al. Endoscopic medial orbital fat decompression for proptosis in type 1 graves orbitopathy. Am J Ophthalmol. 2015;159(2):277-284.

17. O'Donnell NP, Virdi M, Kemp EG. Hertel exophthalmometry: the most appro- priate measuring technique. $\mathrm{Br} J$ Ophthalmol 1999; 83:1096B

18. Leong SC, Karkos PD, Macewen CJ, White PS. A systematic review of outcomes following surgical decompression for dysthyroid orbitopathy. Laryngoscope. 2009;119(6):1106-1115.

19. Walsh TE, Ogura JH. Transantral orbita decompression for malignant exophthal- mos. Laryngoscope. 1957 Jun;67(6):544-68.

20. Leong SC, White PS. Outcomes follow ing surgical decompression for dysthyroid orbitopathy (Graves' disease). Curr Opin Otolaryngol Head Neck Surg. 2010 Feb;18(1):37-43.

21. Metson R, Dallow RL, Shore JW. Endoscopic orbital decompression. Laryngoscope. 1994;104(8 Pt 1):950-957.

22. Metson R, Samaha M. Reduction of diplopia following endoscopic orbital decompression: the orbital sling technique. Laryngoscope. 2002;112(10):1753-1757.

23. Wright ED, Davidson J, Codere F, Desrosiers M. Endoscopic orbital decompression with preservation of an inferomedial bony strut: minimization of postoperative diplopia. J Otolaryngol. 1999 Oct;28(5):252-6.

24. Shepard KG, Levin PS, Terris DJ. Balanced orbital decompression for Graves' ophthalmopathy. Laryngoscope 1998;108:1648-53.
25. Graham SM, Brown $C L$, Carter KD, Song $A$, Nerad JA. Medial and lateral orbital wall surgery forbalanced decompression in thyroid eye disease. Laryngoscope 2003:113:1206-9.

Nikolaos Tsetsos MD, MSc

Otorhinolaryngology

Head and Neck Surgery Department

424 General Military Hospital

Thessaloníki

Greece

Tel: +30 2310381736,

$+306978953761$

Fax: +30 2310381010

E-mail: tsetsosnikos@yahoo.gr 